\title{
La nueva realidad: Manejo endovascular del trauma de carótida
}

\author{
The new era: Endovascular approach for carotid artery trauma
}

\author{
Carlos Andrés Serna-Arbeláez ${ }^{1 D}$, Luis Saldarriaga ${ }^{1}$ D, Edgar Folleco ${ }^{2}$, Alberto L. Rosero², \\ Yaset Caicedo $^{3} \mathbb{D}$, Sofia Daniela Timaran ${ }^{4}$, Jose Julián Serna ${ }^{5,6} \mathbb{D}$, Alberto García ${ }^{5,6} \mathbb{D}$, \\ Fernando Rodríguez-Holguín ${ }^{6}$ D, Carlos A. Ordoñez ${ }^{5,6}$ (D)
}

1 Médico, especialista en Cirugía general; fellow, Cirugía de Trauma y Emergencias, Universidad del Valle. Cali, Colombia.

2 Médico, especialista en Radiología, Servicio de Neuro radiología, Departamento de Radiología, Fundación Valle del Lili, Cali, Colombia.

3 Médico, Centro de Investigaciones Clínicas, Fundación Valle del Lili, Cali, Colombia.

4 Estudiante de medicina, Facultad Ciencias de la Salud, Universidad ICESI, Cali, Colombia.

5 Médico, especialista en Cirugía general y en Cirugía de Trauma y Emergencias. Departamento de Cirugía General, Hospital Universitario del Valle Evaristo García, Universidad del Valle, Cali, Colombia.

6 División de Cirugía de Trauma y Emergencias, Departamento de Cirugía General, Fundación Valle del Lili, Cali, Colombia.

\section{Resumen}

Introducción. La mayoría de las lesiones de carótida cervical en nuestra institución se manejan por vía endovascular. El objetivo de este estudio fue describir los desenlaces del manejo de las lesiones de carótida cervical en un hospital de cuarto nivel en la ciudad de Cali, Colombia.

Métodos. Estudio de series de casos, retrospectivo, descriptivo, en pacientes con trauma de carótida (penetrante y cerrado), admitidos en un centro de alta complejidad de la ciudad de Cali, en el periodo comprendido desde enero de 2018 hasta enero de 2020.

Resultados. Se evaluaron 20 pacientes con lesión de carótida, de los cuales 90 \% tenía trauma penetrante, en su mayoría por proyectil de arma de fuego. La zona más frecuentemente afectada fue la carótida interna (65 \%) y el 40 \% de los pacientes presentaban síntomas neurológicos al ingreso. Se realizó manejo endovascular en 13 pacientes, con un $75 \%$ de éxito en el manejo endovascular al ingreso. La mortalidad general fue del $20 \%$, que en su mayoría estuvo relacionada con traumatismo en otros órganos. El $69 \%$ de los pacientes quedaron sin secuelas neurológicas al alta y el $25 \%$ con secuelas mínimas.

Discusión. Se muestra una serie de casos con lesión de carótida donde, teniendo en cuenta las variables de mal pronóstico para hacer una selección adecuada de los pacientes candidatos a este tipo de terapia, el resultado del manejo endovascular fue exitoso.

Palabras clave: traumatismos de las arterias carótidas; heridas y traumatismos; traumatismo múltiple; angiografía; procedimientos endovasculares; embolización terapéutica.

Fecha de recibido: 15/09/2020 - Fecha de aceptación: 15/11/2020 - Fecha de publicación en línea: 06/04/2021 Correspondencia: Carlos A. Ordoñez, MD, FACS. Carrera 98 \#18-49, Fundación Valle del Lili, Cali, Colombia. Teléfono: 300631 9118. Correo electrónico: ordonezcarlosa@gmail.com

Citar como: Serna-Arbeláez CA, Saldarriaga L, Folleco E, Rosero AL, Caicedo Y, Timaran SD, Serna JJ, García A, Rodríguez-Holguín F, Ordoñez CA. La nueva realidad: Manejo endovascular del trauma de carótida. Rev Colomb Cir. 2021;36:421-6. https://doi.org/10.30944/20117582.832

Este es un artículo de acceso abierto bajo una Licencia Creative Commons - BY-NC-ND https://creativecommons.org/licenses/by-ncnd/4.0/deed.es 


\begin{abstract}
Introduction. Most cervical carotid injuries in our institution are managed by endovascular approach. The objective of this study was to describe the outcomes of the endovascular management of cervical carotid lesions in at a I Level Trauma Center in Cali, Colombia.

Methods. Retrospective, descriptive case series study in patients with both penetrating and blunt carotid trauma who were admitted to a I Level Trauma Center between January 2018 and January 2020.

Results. Twenty patients with carotid injury were evaluated, of which $90 \%$ had penetrating trauma, mostly from a firearm projectile. The most frequently affected area was the internal carotid (65\%) and $40 \%$ of the patients had neurological symptoms on admission. Endovascular management was performed in 13 patients, with a $75 \%$ success rate in endovascular management on admission. Overall mortality was $20 \%$, most of which was related to trauma to other organs; $69 \%$ of the patients were left without neurological sequelae at discharge and $25 \%$ with minimal sequelae.
\end{abstract}

Discussion. We describe a case series of patients with cervical carotid injury, taking into account the variables of poor prognosis to make an adequate selection of patients for endovascular management, the result of endovascular management was successful.

Keywords: carotid artery injuries; wounds and injuries; multiple trauma; angiography; endovascular procedures; therapeutic embolization.

\section{Introducción}

Las lesiones de carótida han sido reportadas con una frecuencia del $6 \%$ entre las lesiones en cuello y un $22 \%$ de las vasculares ${ }^{1-5}$. Se ha descrito que el $80 \%$ de los pacientes con trauma penetrante de carótida presentan choque hemorrágico al ingreso, el $60 \%$ sangrado activo, el $33 \%$ hematoma y el $20 \%$ signos de déficit neurológico ${ }^{2}$. Además de una alta tasa de mortalidad, los pacientes también tienen alto riesgo de secuelas neurológicas si sobreviven, con una tasa de hasta un $70 \%$ de infarto cerebral ${ }^{6}$.

En la actualidad, el manejo del trauma de carótida es un desafío clínico ${ }^{7,8}$. Clásicamente se había propuesto un abordaje quirúrgico de urgencia para el tratamiento de este tipo de lesiones, con resultados poco satisfactorios. Por eso, el uso de tecnología con angiografía que permite el intervencionismo vascular, es una nueva propuesta para el manejo, con experiencias favorables, tanto en trauma cerrado como penetrante ${ }^{4-6}$. En una serie de casos del Hospital San Vicente de Paul, en Medellín, Colombia, se reportó un éxito del 97 \% con el manejo endovascular de las lesiones de carótida ${ }^{7}$.

El paciente candidato a manejo endovascular se ha descrito como aquel hemodinámicamente estable, cuyo estado clínico permite el estudio por angiotomografía, para definir la mejor intervención ${ }^{8,9}$. Los factores al momento de definir el riesgo de este tipo de lesiones, en el paciente con trauma de carótida, son el tipo y la localización de la lesión ${ }^{10}$.

El objetivo de este estudio fue presentar la experiencia respecto al manejo de trauma de carótida, de un centro de IV nivel de atención, con especial énfasis en los pacientes sometidos a manejo endovascular.

\section{Métodos}

Estudio de serie de casos con registro retrospectivo, de pacientes atendidos en la Fundación Valle del Lili, un centro de alta complejidad y de referencia para el manejo del paciente con trauma, en Cali, Colombia.

Se incluyeron pacientes con edad mínima de 18 años y trauma de carótida, diagnosticado por imagen o por exploración quirúrgica, desde enero de 2018 hasta enero de 2020. Se excluyeron todos los pacientes menores de 18 años, con trauma craneoencefálico severo (AIS > 3), o quienes fallecieron al ingreso en el servicio de urgencias. 
Los pacientes se identificaron a partir de los códigos CIE-10 en el sistema de información de la institución, se revisaron los criterios de selección en la historia clínica y, en quienes se confirmó trauma de carótida, se realizó un nuevo análisis imagenológico por un radiólogo independiente. Como resultado de ello, los pacientes fueron reclasificados en el grado del trauma y se procedió a registrar los datos de interés para el estudio en una base de datos de Microsoft Excel.

Se evaluaron las variables que incluyeron datos demográficos, edad, tipo trauma, tipo de lesión, ubicación de la lesión, índice de severidad en trauma, índice de choque, manejo y trasfusiones sanguíneas. Se hizo seguimiento de la evolución clínica durante los primeros 30 días de ingreso y se evaluaron el resultado neurológico y la mortalidad (por cualquier causa durante la estancia hospitalaria).

Para el análisis descriptivo se reportaron las variables categóricas, como frecuencias relativas y absolutas, y las variables continuas, con mediana y rango intercuartílico (RIQ). El análisis estadístico se realizó usando Lenguaje R versión 3.6.3 ${ }^{11}$.

\section{Resultados}

En la base de datos de la institución se identificaron 28 pacientes que cumplían los criterios de inclusión, y se hizo una revisión exhaustiva de la historia clínica y las imágenes diagnósticas, encontrando que 20 pacientes realmente cumplían los estándares del estudio y fueron incluidos en el análisis. Las características de los pacientes están resumidas en la tabla 1.

El $90 \%$ de los pacientes eran de sexo masculino, el mecanismo más frecuente fue el trauma penetrante ( $90 \%$ ), y de estos, el $80 \%$ fueron por proyectil de arma de fuego. El $95 \%$ de los pacientes presentaron algún grado de trauma craneoencefálico y la escala de Coma de Glasgow tuvo una mediana de 14 (RIQ: 9-15). El $40 \%$ de los pacientes ingresaron con paresia en alguna extremidad, el $65 \%$ con sangrado activo por la herida traumática, el $40 \%$ con hematoma expansivo y el $55 \%$ con hematoma estable. El $50 \%$ de los pacientes requirió trasfusión de productos sanguíneos al ingreso.
Tabla 1. Experiencia del manejo en pacientes con trauma de carótida

\begin{tabular}{|c|c|}
\hline \multicolumn{2}{|c|}{ Pacientes con trauma de carótida $(n=20)$} \\
\hline \multicolumn{2}{|c|}{ Genero } \\
\hline Masculino & $18(90 \%)$ \\
\hline \multicolumn{2}{|l|}{ Tipo de Trauma } \\
\hline Penetrante & $18(90 \%)$ \\
\hline Herida por arma cortopunzante & $2(10 \%)$ \\
\hline Herida por proyectil de arma de fuego & $16(80 \%)$ \\
\hline Cerrado & $2(10 \%)$ \\
\hline \multicolumn{2}{|l|}{ Signos vitales al ingreso } \\
\hline Frecuencia Cardiaca, Ipm & $97(70-117)$ \\
\hline Escala de Coma de Glasgow & $14(9-15)$ \\
\hline Presión Arterial Sistólica, mmHg & $117(80-125)$ \\
\hline Paresia al ingreso & $8(40 \%)$ \\
\hline Sangrado & $13(65 \%)$ \\
\hline Hematoma expansivo & $8(40 \%)$ \\
\hline Hematoma estable & $11(55 \%)$ \\
\hline Transfusión & $10(50 \%)$ \\
\hline Trauma craneoencefálico & $19(95 \%)$ \\
\hline \multicolumn{2}{|l|}{ Abordaje quirúrgico } \\
\hline Rafia & $1(5 \%)$ \\
\hline Injerto de safena & $1(5 \%)$ \\
\hline Ligadura & $1(5 \%)$ \\
\hline \multicolumn{2}{|l|}{ Arteriografía } \\
\hline Diagnóstica & $6(40 \%)$ \\
\hline Terapéutica & $9(60 \%)$ \\
\hline Diagnóstica & $6(40 \%)$ \\
\hline \multicolumn{2}{|l|}{ Manejo endovascular } \\
\hline Arteriografía (diagnóstica) & $6(40 \%)$ \\
\hline Embolización & $2(13 \%)$ \\
\hline Stent & $7(47 \%)$ \\
\hline \multicolumn{2}{|l|}{ Porción de la Carótida } \\
\hline Común & $5(25 \%)$ \\
\hline Externa & $2(10 \%)$ \\
\hline Interna & $13(65 \%)$ \\
\hline \multicolumn{2}{|l|}{ Tipo de Lesión } \\
\hline Disección menor del 30 \% & $2(10 \%)$ \\
\hline Disección mayor del 30 \% & $1(5 \%)$ \\
\hline Pseudoaneurisma & $7(35 \%)$ \\
\hline Ruptura & $3(15 \%)$ \\
\hline Amputación & $7(35 \%)$ \\
\hline \multicolumn{2}{|l|}{ Terapia antiagregante / Anticoagulación } \\
\hline No & $5(25 \%)$ \\
\hline Antiagregación plaquetaria & $7(35 \%)$ \\
\hline Anticoagulación & $1(5 \%)$ \\
\hline Combinado & $7(35 \%)$ \\
\hline \multicolumn{2}{|c|}{ Compromiso neurológico al alta (vivos $n=16$ ) } \\
\hline Ninguno & $11(69 \%)$ \\
\hline Menor & $4(25 \%)$ \\
\hline Mayor & $1(6 \%)$ \\
\hline \multicolumn{2}{|l|}{ Resultados Clínicos } \\
\hline Estancia hospitalaria, días & $5(5-14)$ \\
\hline Arresto cardiaco & $5(25 \%)$ \\
\hline Mortalidad & $4(20 \%)$ \\
\hline
\end{tabular}


Las lesiones se localizaron en carótida común (25\%), carótida externa (10 \%) y carótida interna (65\%), y por el tipo de lesión, se clasificaron como disección de la íntima (15\%), pseudoaneurisma (35\%), ruptura con sangrado activo (15\%) y amputación del vaso (35 \%).

Cinco pacientes no se llevaron a arteriografía, de los cuales, dos murieron en urgencias antes de cualquier intervención y tres fueron sometidos a cirugía inmediata. De los que fueron a cirugía, todos tenían lesión de arteria carótida común: en uno se realizó rafia de la lesión por proyectil de arma de fuego, en uno se colocó un injerto invertido de safena, y el otro paciente requirió ligadura emergente por el contexto del trauma, falleciendo posteriormente.

Quince pacientes se llevaron a arteriografía carotidea; $60 \%$ fueron terapéuticas y el resto fueron diagnósticas pues no requirieron intervenciones adicionales. De las arteriografías terapéuticas, en dos pacientes se hizo "embolización" de la arteria carótida interna, uno por estar ocluida completamente y otro por tener polígono de Willis permeable con sangrado activo en porción petrosa; siete pacientes requirieron manejo con stent recubierto, dos puestos en la carótida común y cinco en la porción cervical de la carótida interna (tabla 2).

Ninguno de los pacientes que se llevaron a cirugía o murieron recibió manejo antiagregan- te o anticoagulación, mientras que todos los pacientes sometidos a intervencionismo vascular recibieron alguna de estas terapias después del procedimiento.

En total murieron cuatro pacientes, dos por trauma múltiple durante la atención en urgencias, uno por sangrado masivo en diferentes sitios (al paciente a quien se ligó carótida) y otro por accidente cerebro vascular (ACV) isquémico instaurado previo al ingreso (en quien se "embolizó" la carótida interna pues ya estaba instaurado el daño neurológico). La mortalidad general fue del $20 \%$ y se presentó paro cardiaco en 5 pacientes, de los cuales uno sobrevivió.

De los 16 pacientes que sobrevivieron, uno (6\%) quedó con secuelas neurológicas mayores (encefalopatía hipóxico-isquémica), cuatro (25\%) con secuelas neurológicas menores (paresia o praxia) y 11 (69\%) pacientes no tuvieron ninguna secuela neurológica. La estancia hospitalaria promedio fue de 5 días (RIQ: 5-14).

\section{Discusión}

Cuando estudiamos el trauma de carótida, en la literatura encontramos más que todo publicaciones de serie de casos, pues al ser una lesión poco frecuente y con consecuencias devastadoras, es poco probable que se puedan hacer estudios con intervenciones comparativas, de buena calidad epidemiológica.

Tabla 2. Pacientes con trauma de carótida interna manejados con stent

\begin{tabular}{lccccc}
\hline Caso & $\mathbf{1}$ & $\mathbf{2}$ & $\mathbf{3}$ & $\mathbf{4}$ & $\mathbf{5}$ \\
\hline Edad, años & 42 & 29 & 42 & 25 & $\mathbf{4}$ \\
\hline \begin{tabular}{l} 
Tipo de herida \\
\hline $\begin{array}{l}\text { Puntaje en la escala } \\
\text { de Glasgow al ingreso }\end{array}$
\end{tabular} & $\begin{array}{c}\text { Proyectil de arma } \\
\text { de fuego }\end{array}$ & $\begin{array}{c}\text { Proyectil de arma } \\
\text { de fuego }\end{array}$ & $\begin{array}{c}\text { Proyectil de arma } \\
\text { de fuego }\end{array}$ & $\begin{array}{c}\text { Proyectil de arma } \\
\text { de fuego }\end{array}$ & $\begin{array}{c}\text { Proyectil de arma } \\
\text { de fuego }\end{array}$ \\
\hline $\begin{array}{l}\text { Sangrado activo } \\
\text { Tipo de lesión }\end{array}$ & $\mathrm{Si}$ & 15 & 13 & 15 & 15 \\
\hline $\begin{array}{l}\text { Compromiso } \\
\text { neurológico al alta }\end{array}$ & Ninguno & $\mathrm{Si}$ & $\mathrm{Si}$ & $\mathrm{No}$ & $\mathrm{Si}$ \\
\hline $\begin{array}{l}\text { Estancia hospitalaria, } \\
\text { días }\end{array}$ & 6 & Leve & Ninguno & Leve & Ninguno \\
\hline
\end{tabular}


En los últimos 80 años ha cambiado mucho el enfoque de este tipo de trauma y, sobre todo en los últimos 20 años, se han ido refinando métodos diagnósticos y terapéuticos propuestos por las diferentes asociaciones, pero aun es difícil lograr un consenso claro, pues existen muchas variables a controlar, y cada una de esas variables puede influir fuertemente en los desenlaces neurológicos del paciente ${ }^{12}$.

Hasta hace 3 décadas, todo paciente con sospecha de lesión vascular en el cuello tenía indicación de intervención quirúrgica emergente. Aunque esta premisa aún se mantiene, se ha dado cabida al manejo endovascular en casos específicos ${ }^{4}$.

$\mathrm{Al}$ evaluar un paciente con lesión de arteria carótida es importante tener en cuenta el sitio anatómico, tiempo de evolución de la lesión, mecanismo del trauma, escala de coma de Glasgow, presencia de síntomas neurológicos, estabilidad hemodinámica, clasificación de la lesión vascular, disponibilidad de quirófano hibrido, otras lesiones asociadas que amenazan la vida y capacidad de manejo en unidad de cuidado intensivo (UCI). Con todas estas variables resueltas, se debe tomar la decisión de "revascularizar" o no el paciente, contemplando a futuro el riesgo conversión a un ACV hemorrágico, edema cerebral y muerte por reperfusión o, por el contrario, mejoría neurológica por perfusión de zonas con penumbra isquémica ${ }^{12,13}$.

En nuestro trabajo encontramos que casi todas las lesiones eran penetrantes, por proyectil de arma de fuego, y la porción más frecuentemente afectada fue la carótida interna, lo cual confiere un peor pronóstico neurológico. Pero, por otro lado, podemos mostrar que, a pesar de esto, el manejo endovascular fue exitoso en la mayoría de los casos, con mínimo compromiso neurológico al alta en los pacientes que sobrevivieron, convirtiendo esta intervención en una muy buena opción para el manejo de pacientes con lesión de arteria carótida que se encuentran hemodinámicamente estables.

La mortalidad en nuestro estudio estuvo más asociada a la presencia de otras lesiones corporales que a la intervención vascular por sí misma, pero esto no quiere decir que el manejo endovascular este exento de complicaciones, por eso es tan importante evaluar el contexto del paciente y sus variables de mal pronóstico (como tiempo de lesión o síntomas neurológicos) antes de decidir si "revascularizar" o no el cerebro 4 .

Este estudio posee múltiples limitaciones, pues al ser un estudio retrospectivo descriptivo su peso estadístico es limitado; además, por las características del centro de trauma donde se realizó el estudio, probablemente allí fueron trasladados los pacientes con lesiones más graves, y de ahí radica la desproporción de pacientes con trauma penetrante versus trauma cerrado, y el compromiso frecuente de la arteria carótida interna.

\section{Conclusión.}

En este estudio se muestra una serie de casos donde el manejo endovascular fue exitoso, teniendo en cuenta siempre las variables de mal pronóstico para hacer una selección adecuada de los pacientes candidatos a este tipo de terapia. Este trabajo puede servir de punto de partida para futuros estudios de mayor poder estadístico, que nos ayuden a tomar buenas decisiones con respecto al manejo del trauma de arteria carótida cervical, pues en la actualidad la bibliografía es limitada.

\section{Cumplimiento de normas éticas}

Consentimiento informado: Este es un estudio retrospectivo descriptivo de revisión de historias clínicas, y como tal, no hay necesidad de un consentimiento informado. Se obtuvo aprobación por el Comité de Ética de la institución para llevar a cabo la investigación.

Conflicto de interés: Ninguno declarado por los autores. Financiación: Los recursos de financiación del proyecto provienen en su totalidad de aportes de los autores.

\section{Declaración de contribución de los autores.}

- Concepción y diseño del estudio: Carlos Serna, Edgar Folleco, Alberto Rosero, Yaset Caicedo, Jose Serna, Alberto García, Carlos Ordoñez.

- Adquisición de datos: Carlos Serna, Luis Saldarriaga, Edgar Folleco, Alberto Rosero, Yaset Caicedo, Sofia Timaran, Jose Serna.

- Análisis e interpretación de datos: Yaset Caicedo, Carlos Serna, Jose Serna, Alberto García, Fernando Rodríguez, Carlos Ordoñez.

- Redacción del manuscrito: Carlos Serna, Yaset Caicedo, Jose Serna, Luis Saldarriaga, Sofia Timaran. 
- Revisión crítica: Carlos Serna, Yaset Caicedo, Jose Serna, Edgar Folleco, Alberto Rosero, Alberto García, Fernando Rodríguez, Carlos Ordoñez

\section{Referencias}

1. Navsaria P, Omoshoro-Jones J, Nicol A. An analysis of 32 surgically managed penetrating carotid artery injuries. Eur J Vasc Endovasc Surg 2002;24:349-55. https://doi.org/10.1053/ejvs.2002.1736.

2. Demetriades D, Asensio JA, Velmahos G, Thal E. Complex problems in penetrating neck trauma. Surg Clin North Am 1996;76:661-83. https://doi.org/10.1016/S0039-6109(05)70475-8.

3. Lee TS, Ducic Y, Gordin E, Stroman D. Management of Carotid Artery Trauma. Craniomaxillofac Trauma Reconstr 2014;7:175-89. https://doi.org/10.1055/s-0034-1372521.

4. Biagioni RB, Burihan MC, Nasser F, Biagioni LC, Ingrund JC. Endovascular treatment of penetrating arterial trauma with stent grafts. Vasa - Eur J Vasc Med 2018;47:125-30. https://doi.org/10.1024/0301-1526/a000672.

5. Foreman PM, Harrigan MR. Blunt Traumatic Extracranial Cerebrovascular Injury and Ischemic Stroke. Cerebrovasc Dis Extra 2017;7:72-83. https://doi.org/10.1159/000455391.

6. Kraus RR, Bergstein JM, Debord JR. Diagnosis, treatment, and outcome of blunt carotid arterial injuries. Am J Surg 1999;178:190-3. https://doi.org/10.1016/S0002-9610(99)00157-9.

7. Jiménez HC, Romero E, Medina R, Botache W, Sanjuán J, Martínez C, et al. Caracterización de trauma arterial periférico en un hospital de tercer nivel. Rev Colomb Cirugía 2018;33:272-9.

https://doi.org/10.30944/20117582.72.

8. Pacheco MA, Aldana GE, Granados ÁE, Martínez LE, Santacoloma J, Baquero RL, et al. Manejo del trauma penetrante de cuello en dos hospitales de Bogotá, Colombia. Rev Colomb Cirugía 2018;33:34-43. https://doi.org/10.30944/20117582.45.

9. Herrera DA, Vargas SA, Dublin AB. Endovascular treatment of penetrating traumatic injuries of the extracranial carotid artery. J Vasc Interv Radiol 2011;22:28-33. https://doi.org/10.1016/j.jvir.2010.09.022.

10. Hundersmarck D, Reinders Folmer E, de Borst GJ, Leenen LPH, Vriens PWHE, Hietbrink F. Penetrating Neck Injury in Two Dutch Level 1 Trauma Centres: the Non-Existent Problem. Eur J Vasc Endovasc Surg 2019;58:455-62. https://doi.org/10.1016/j.ejvs.2019.04.020.

11. R Core Team. R: A language and environment for statistical computing. 2019.

12. Blitzer DN, Ottochian M, O'Connor J, Feliciano D V., Morrison JJ, DuBose JJ, et al. Penetrating Injury to the Carotid Artery: Characterizing Presentation and Outcomes from the National Trauma Data Bank. Ann Vasc Surg 2020:1-8. https://doi.org/10.1016/j.avsg.2020.03.013.

13. Martin MJ, Mullenix PS, Steele SR, Asensio JA, Andersen CA, Demetriades D, et al. Functional outcome after blunt and penetrating carotid artery injuries: Analysis of the National Trauma Data Bank. J Trauma - Inj Infect Crit Care 2005;59:860-4.

https://doi.org/10.1097/01.ta.0000187964.47703.e9. 